\title{
DESIGN AND RESEARCH OF SERVICE PLATFORM FOR PROTECTION AND DISSEMINATION OF CULTURAL HERITAGE RESOURCES OF THE SILK ROAD IN THE TERRITORY OF CHINA
}

\author{
Lie Zhang ${ }^{\mathrm{a}}$, Wen Zhang ${ }^{\mathrm{a}}$, Shijing Zeng ${ }^{\mathrm{a}}$, Wen Na ${ }^{\mathrm{a}}$, Hong Yang ${ }^{\mathrm{a}}$, Jin Huang ${ }^{\mathrm{b}}$, Xingdong Tan ${ }^{\mathrm{c}}$, Zhijun Sun ${ }^{\mathrm{d}}$ \\ a Academy of Art\& Design, Tsinghua University, Room. B466, Academy of Art\& Design, Tsinghua University, Beijing, China-(zhlie, \\ vanezh, nw11,csj13, yh678)@tsinghua.edu.cn \\ b Tencent, Inc. Shenzhen, China-jinhuang@tencent.com \\ c EZ360 Information Technology Co., Ltd., Beijing, China-tanxindong@ez360.cn \\ d Dunhuang Academy Network\& Information Center, Dunhuang, China-sunzj@dha.ac.cn
}

KEY WORDS: Silk Road, Cultural heritage, Dissemination design, Service design, Network platform

\begin{abstract}
:
The Silk Road, a major traffic route across the Eurasia continent, has been a convergence for the exchange, communication and dissemination of various cultures such as nations, materials, religions and arts for more than two thousand years. And the cultural heritage along the long and complicate route has been also attractive. In recent years, the Silk Road - the Road Network along the Chang'an-Tianshan Mountain has been listed in the Directory of World Cultural Heritage. The rare and rich cultural resources along the Silk Road, especially those in the territory of China, have attracted attentions of the world.

This article describes the research ideas, methods, processes and results of the planning design on the internet-based dissemination services platform system for cultural heritage resources. First of all, it has defined the targeting for dissemination services and the research methods applied for the Silk Road heritage resources, based on scientific and objective spatial measurement and research on history and geography, to carry on the excavation of values of cultural resource for the target users. Then, with the front-end art exhibit by means of innovative IT, time and space maps of cultural heritage resources, interactive graphics display, panoramic three-dimensional virtual tour, and the Silk Road topics as the main features, a comprehensive and multi-angle cultural resources dissemination services platform is built. The research core of the platform is a demand-oriented system design on the basis of cultural resources and features as the fundamental, the value of contemporary manifestation as the foundation, and cultural dissemination and service as a starting point. This platform has achieved, temporal context generalization, interest profiles extension, online and offline adaptation, and other prominent innovations. On the basis of routes heritage resource protection and dissemination services with complex relationship between time and space, and the Silk Road as the representative, practice and research of the platform in the internet context help to provide an application reference and theoretical basis.
\end{abstract}

\section{INTRODUCTION}

\subsection{Motivation}

The Silk Road, first appeared in the second century BC, was a major traffic route linking China and Eurasia. From Chang'an and Luoyang, the two Chinese ancient capitals, to the Mediterranean countries, ancient Chinese civilization, Indian civilization, Persian civilization and other cultures have been converged, exchanged, integrated, and communicated along the Silk Road, which have played a profound role in promoting communication of religion, art and technology as well as the exchange of materials between various civilizations, and have left a large number of rich cultural heritages. These cultural heritages include cave temples, ancient towns, tombs, and many fantastic murals, sculptures, Cultural relics, etc., which reflect the historical period of the magnificent cultural convergence and dissemination, with tremendous value on research and conservation and significant development potential.

\subsection{Goals}

This article elaborates, design of internet-based dissemination services platform of heritage resources of the Silk Road, as part of the relevant national topics by Tsinghua University and the Dunhuang Research Institute and a number of academic institutions. With the goal of the establishment of an open, systematic, scientific, and efficient cultural dissemination and services platform system, on the basis of results of technical innovations on related issues, the study is combined with interactive information design, to try to dig, display, distribute with multi-angle, all-round, and in-depth manner and to promote the Silk Road's precious cultural heritage resources and its splendid culture, and to contribute to the promotion of cultural, social, economic exchanges and development of the areas along the Silk Road. This study is, for all types of researchers, enthusiasts and ordinary tourists at home and abroad, who concern the Silk Road, to provide cultural resources study and inquiry, virtual tour, reservation service, exchange and share of experiences, and other functions, such as geography and history information system display of cultural heritage resources based on the time and space dimensions, virtual tour with panoramic and three-dimensional technology, appreciation of cultural heritage by HD image data, customized "My Silk Road" information services and sharing, etc.

\section{RELATED WORKS}

In recent years, there are numerous website platforms and applications on the protection and dissemination of cultural 
heritages, which have been involved in wider and wider fields, and their quality and cultural depth have also been constantly improved. For example, the National Institute of Informatics of Japan has started to implement the "Digital Silk Road program" for scholars since 2003, see http://dsr.nii.ac.jp/senga/index.html. Currently, the project has launched a digital Silk Road website, including Chinese grotto data sources such as the Dunhuang Mogao Grottoes, the Bozi Creek Caves and the Kizil Grottoes, and the contents consist of grotto number table, the master grotto elevation, and related webpages. In 2014, the "History and Geography Information System of the Silk Road" developed by Institute of Geography and History of Fudan University was launched online. The system accurately presents the Xuanzang route, the Kalaqi ancient route, etc., demonstrating academic achievements of the institute with long-term research and study on the routes of the Silk Road, see http://sc.youdell.com.The Dunhuang Academy makes cooperation with domestic and foreign academic institutions in a long run, they have achieved fruitful results in digitization research of the Dunhuang cultural heritage, which have been applied and published in a number of professional academic websites. In addition, there have been emerged many public websites and mobile applications for the dissemination of cultural heritages of the cities with very concentrated cultural resources, such as Dunhuang, Xi'an, Lanzhou, Urumqi, etc.. These websites and applications promotes cultural knowledge of the Silk Road, deepens awareness and understanding of people, narrows the distance between the people of each area and the distant and long Silk Road.

Meanwhile, with the overall promotion on the understanding of the value of cultural heritage resources of the Silk Road, we also realize that there are many deficiencies on the existing digital communication service platforms, on the aspects of cultural characteristics, historical background, and regional culture of the Silk Road. There are a lot of room for improvement on systematic and scientific macro planning, depth and richness of content mining, advanced technology, and usability and experience design on the attractiveness of the public, etc.

\section{THE BASIC IDEAS AND METHODS}

\subsection{Core content}

The study is first of all based on scientific and objective measurement of geography and space, high-precision digital data acquisition and literature research, to conduct in-depth, targeted, space and historical research, and multidimensional value mining. By combining with behaviour characteristics analysis for meeting dissemination and the audiences, it can play technical expertise of modern digital means of communication to form communication service platform and application system with unique and practical value.

\subsection{The basic ideas}

In the grasp of the core content of the project, we think that the object of study is the issue of protection and dissemination of cultural resources and sustainable use with a huge space and time frame, its guiding ideology should have the following two points:

First, it is to explore the value based on the cultural heritage resources and characteristics. The Silk Road, lasting for thousands of years, across the Eurasian continent, is a magnificent and unique historical picture in the history of human civilization. Its current situation of relics and historical origin attract people to pursue, in addition, its stories in history and the contexts, phenomena and comparisons of social, cultural and artistic changes presented in the stories are also unique charm of the Silk Road. Full understand, excavation and combing of the characteristics of these cultural resources are undoubtedly a fundamental standpoint for communication and service.

Second, it is demand-oriented system design based on the contemporary value manifestation, with cultural communication and service as a starting point. How to make contemporary value of cultural heritage to be fully reflected (Dodsworth, 2001)? Which requires us to have a sense of service concepts and human feelings, and carry out an in-depth study of the relationship between cultural relics and interests, behaviour, expectations of the audiences. The different audience groups with different needs have various interest and attention on the type and level of culture; in different situations, people's behaviour patterns and transmission of cultural information have different characteristics. Therefore, the basic idea of the design of the research work is an effective communicated design system that connects ontology features of cultural resources and crossover studies of the audiences with anthropology features, in an interactive narrative manner that is in accordance with the propagation law.

\subsection{Research Methods}

During the study, we use a combination of systems analysis and scenario analysis. The required data standards of content acquisition and dissemination by system platform, are not only based on the nature of cultural heritage, but also necessary to be combined with people in a variety of features and application scenarios to make repeated deduction; In the architecture design, we need to achieve the harmony among flexible platform, rich content, and demand-oriented target; in addition, it also needs positioning analysis and comprehensive study that should be effectively for the needs of users, so as to achieve efficient cultural communication and excellent services in terms of functionality and interaction design. The overall framework of research ideas is in Figure 1.

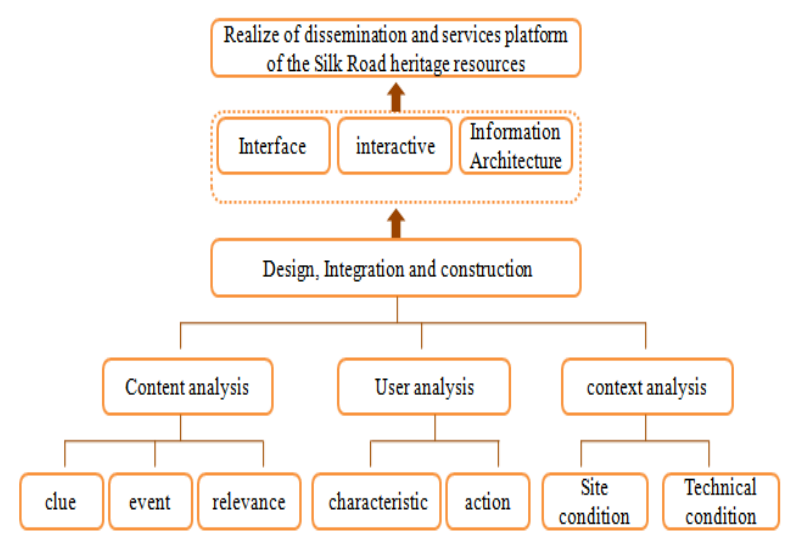

Figure 1: The general research framework of dissemination platform for major cultural resources of the Silk Road

\section{RESULTS OF DESIGN STUDY}

\subsection{Framework and function design}

On based of collection, collation, research and mining value about Silk Road cultural heritage resources, we classified and 
integrated comprehensively. First, the basis-The historical clues, spatial distribution, cultural landscape and other resources, are summarized as historic sites, cultural relics, historical events and folklore, which also constituted a "cultural map" of the body. Second, the special topic-Ignoring the limitations of time and space, we grouped into several thematic city, religion, literature, art, ethnic, etc. These topics were reflected on the "cultural map." Third, Service. Especially information about room and board, which also constitute a "service map." Finally, derivative. Including personalized, experiential and other industries associated, these will play a more prominent role on mobile application development in the later, e.g. Figure 2.

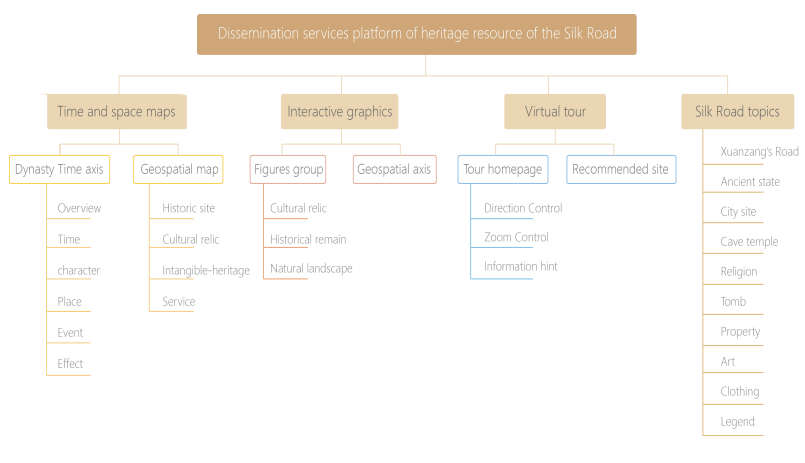

Figure 2: Dissemination services platform information architecture of heritage resource of the Silk Road

\subsection{Information architecture and interaction design}

The basic goal of interaction design is to exhibit rich and varied heritage contents of the Silk Road by means of a clear interactive narrative style so that it is easy for users to understand and use. Based on the previous analysis and deduction, combining with the study of the interaction design process, we construct architectural frame for the interaction and information of the platform, which mainly consists of four parts, space-time maps of cultural heritage, interactive image maps, panoramic three-dimensional virtual tour, and special topics of the Silk Road. This reflects our deep understanding of the spatial and temporal characteristics of the cultural heritages of the Silk Road, understanding of user behaviour and cognitive model as well as innovative technology applications. In addition, it also corresponds to the four different ways and means that uses virtually explore the Silk Road through the network platform. From 2D to 3D, from the real to the virtual, from the macro to the concrete, the panorama of the Silk Road is displayed with wonderful and rich cultural heritage information, e.g. Figure 3, Figure 4.

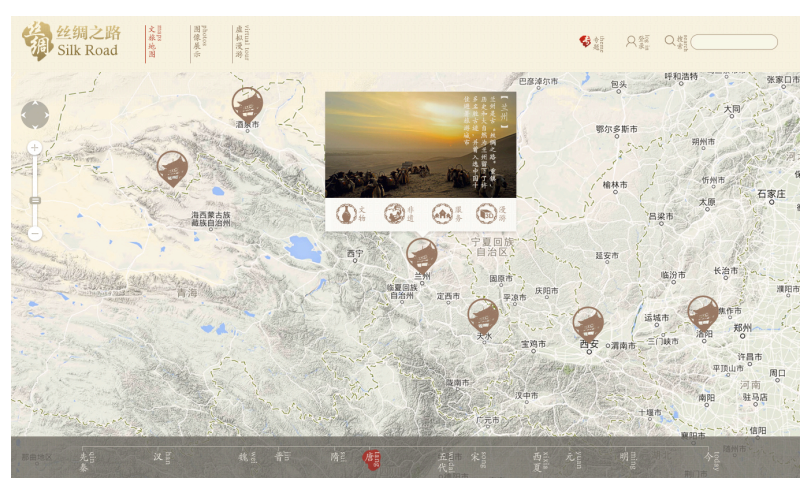

Figure 3: Example on interface design for the Time and space maps of the website

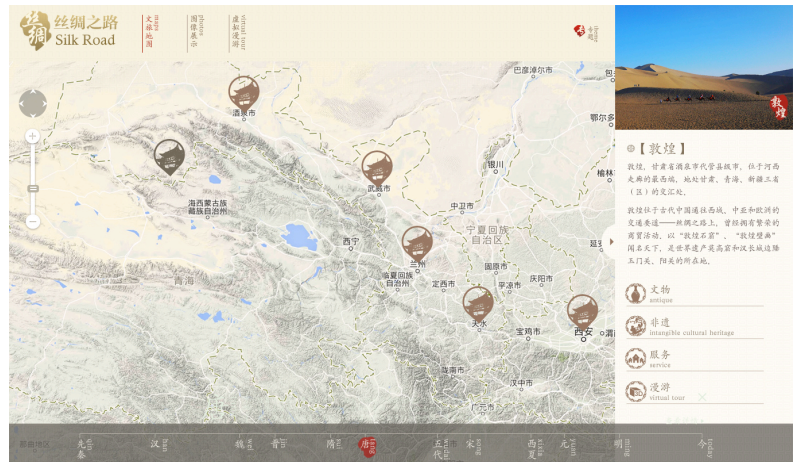

Figure 4: Example on interface design for the Time and space maps with right sidebar

With the space-time maps of cultural heritage, through two dimensions of space maps and interactive time axis, it displays spatial and temporal context, route changes in different historical periods, and other important heritage sites along the Silk Road and other information with panoramic view, which can present comprehensively and visually on the basis of accurate geographic information. With the interactive image maps which combine with the Silk Road, with the geographical feature going through east-west directions, and the current characteristics of human cognition reflected by the so-called "Reading Pictures", the representative landscape photographs of various heritage sites are interactively exhibited according to spatial sequence, so as to convey beautiful images information to attract the users' interest and stimulate desire to explore. The panoramic three-dimensional virtual tour makes use of innovative $3 \mathrm{D}$ reconstruction and interactive technology achievements, in the real three-dimensional visual space, and in the perspective of the audience with immersive experience, giving the audience an immersive visual experience. The special topics of the Silk Road reflect our deep excavation on the cultural value of the Silk Road, by presenting different cultural subjects and contents in a unified geographic information system, the personalized information services can meet the needs of different interest groups, e.g. Figure 5, Figure 6.



Figure 5: Example on interface design for Interactive graphics of the website 


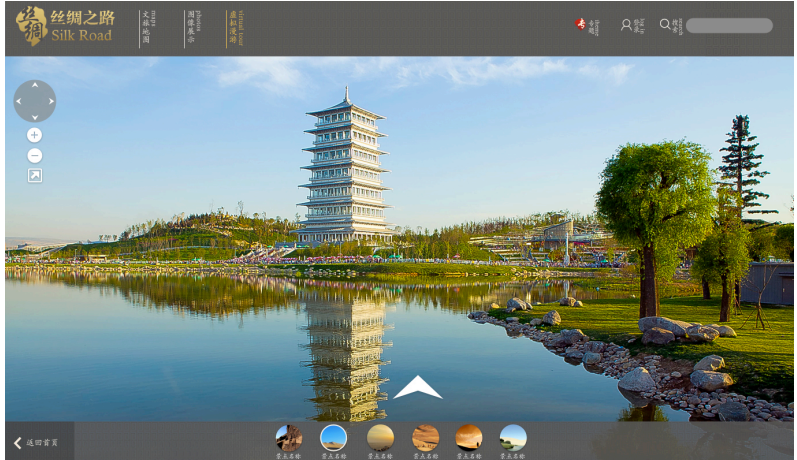

Figure 6: Example on interface design for Three-dimensional virtual tour of the website

\subsection{Interface Design}

Interface design not only needs to meet the requirements of aesthetic and thematic features to be conveyed, but also needs to address the visual recognition, readability, operability of the system, and other issues. The finalized visual interface style integrates into colour and visual elements with the traditional China's charm and the characteristics of the Silk Road in a concise style, so that it can not only demonstrate the vicissitudes and heavy feeling of history, and also pay attention to a sense of modern and minimalist contemporaneity as well as cultural and academic attributes; not only maintain moderate and fine detail and detail design with identifiable characteristics, but also avoid excessive gorgeous to fall into stereotypes.

\subsection{Innovation}

4.4.1 Space-time context: Broad geographic space and a long era of time are prominent features of the Silk Road, while space-time relationship is the easiest model to be understood by people's cognitive psychology. So, it is regarded as a primary means of platform architecture and logical principles, based on the space axis and the time axis, we build space-time dimensions of framework for giving users an intuitive and systematic overall cognitive. In information technology, we seek to precise positioning with GIS, aerial measurement, laser scanning and other means, to rebuild spatial cues of the Silk Road (Guan, 2011). In the study of cultural heritage and historical geography, we strive to determine the specific location and scope of cultural heritage, specific locations, major transportation routes, related cities and passes of major historical events through detailed historical research and archaeological research (Schindler, 2003). Finally, we combine academic achievement of the Silk Road, current geographical information technology, and data acquisition technology to complete construction of the space frame. And we will clarify the context of the time of the Silk Road, grasp the relationship of large span of the historical process and complex or unexpected events, learn the outcome of the current mainstream historians of the Silk Road to complete construction of the time frame. Eventually, platform interactive system with time and space axis as the main feature is formed.
4.4.2 Interest atlas: The cultural content of the Silk Road are vast, broad, and complex, with rich types of cultural heritage, big spatial and temporal span, and multiple levels. Based on the nature of the resources, value considerations, user attention, real state, and other standards, we sort out the cultural contents organically to form clusters of different interests and levels, through a unified data standards and narrative style, so that our system can quickly attract the attention and use by populations with different interests and contexts. For example, for the topics of the Silk Road, we summarize "cave temple", "ancient state", "city sites", "tombs", "art", "property", "clothing", "legend", as well as the famous roads, such as "the Xuan Zang Road", etc. These topics as important cultural heritage on the Silk Road, in the context of time and space location, have been recognized by the academic community, which have also received a high degree of concern in the user survey what we found. Each topic can also correspond to the axis of time and space on cultural resources map, thereby giving a complete understanding, e.g. Figure 7, Figure8.

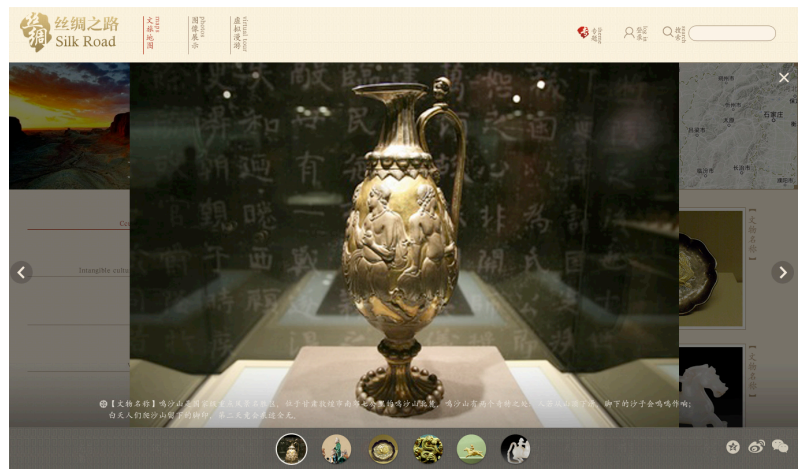

Figure 7: Example of detailed information about cultural relic under "topic" of the website



Figure 8: Example of detailed information about Musical Instruments under "topic" of the website

4.4.3 Online and offline adaptability: Because users have different usage scenarios, online and offline applicability of the platform is an important part for increasing the influence and reflecting the superiority. We use cross-platform technology to carry out adaptive design that is based on the advantages of the characteristics in the application of different platforms. Moreover, combined with augmented reality and other new interactive technology, it achieves the coverage of distinctive contents and services in a longitudinal field, enhances the users' utilization rate and viscosity in order to achieve a higher platform value. 
4.4.4 Method exploration and demonstration: Cultural Route Heritage is a special type of cultural heritage, based on mainly in the road, the water transportation route, which include clear physical boundaries and special dynamic mechanism and historical features, reflect the human interaction, and promote Cultural exchange about the tangible and intangible cultural heritage, have become one or more links across distant geographical space. Cultural route heritage is a organic collection of multi-type cultural heritage. The complexity is evident. Worldwide, there are many cultural sites that distributed by line, ring, or network, such as the Silk Road, the Spice Route in Oman and Israel, the Pilgrimage Route of Santiago in France, and so on. In China, the Grand Canal, Tea-Horse old Road are typical Cultural Route as well. It is a great significance that construction and application of service platform with integration and interaction for research, conservation, propagation of these complex cultural heritages in support of Geographic information systems and huge cultural resource database. Our study on case of the Silk Road made attempts to build a platform that will provide relevant experience in cultural resource integration, framework design, interaction design, etc., meanwhile, we hope to explore more and better methods for protection and propagation about this type of cultural heritage.

\section{CONCLUSION}

The platform proposes an innovative service model to spread the Silk Road heritage through "science and technology support, cultural presentation, space-time architecture, service guide", integrated with Internet and mobile Internet, by means of geographical and historical maps of cultural resources, thematic programs of cultural resources, virtual and digital navigation, and other technical support, in order to provide comprehensive, multi-angle, deep-seated relevant information services of cultural heritage for users, so that users can get in-depth, personalized new experience.

Meanwhile, the platform also proposes static and dynamic models on the time correlation, spatial association, and cultural association of cultural resources of the Silk Road; and builds cultural resource maps in order to provide support with time correlation, spatial association, and cultural association to present for cultural information across the region on the basis of the Internet and mobile Internet services.

Cultural heritage is not only a valuable non-renewable wealth, but also an important resource to highlight the glorious history and culture, and regional characteristics. This service platform, which is for the protection and dissemination of heritage of the Silk Road on the basis of full aware of the importance of value of cultural heritages, with innovative interactive website design and advanced information science and technology, makes efforts for dissemination and development (Zhang, 2013, 2014). We also hope that this platform can attract more cultural heirs and fans so that they can have a new understanding of the Silk Road with full of charm, explore the cultural wealth of the Silk Road that runs through two thousand years and across near thousands of miles, and participate in the career of cultural heritage.

\section{ACKNOWLEDGE}

This article is one of the results of "Cultural tourism service system demonstration based on the Silk Road (China section) major cultural heritage resources" by the National Science and Technology's Support Program (Ministry of science and technology 2011WKYB010).

\section{REFERENCES}

Japan's National Institute of Informatics, 2003. The Plan of Digital Silk Road, http://dsr.nii.ac.jp/senga/index.html Center for Historical Geographical Studies of Fudan University, 2014, Historical Geographic Information System of Silk Road in China, http://sc.youdell.com/

Schindler, K., Grabner, M., Leberl F., 2003. Fast On-Site Reconstruction and Visualization of Archaeological Finds, Proc. 19th International CIPA Symposium.

Dodsworth, C., Mayer, J., 2001. Cultural Heritage and Digital Experience design: Presentation, Adaptation and Competitive Evolution, VAST '01: Proceedings of the 2001 Conference on Virtual reality, Archeology, and Cultural heritage.

Guan, W., You, S., Neumann, U., 2011. GPS-aided Recognition-based User Tracking System with Augmented Reality in Extreme Large-scale Areas. In Proceedings of the second annual ACM conference on Multimedia systems, USC.

Zhang, L., Zhang, W., 2013. Identification and InnovationThe significance of cultural heritages in city culture positioning, City Branding Symposium.

Zhang, L., Zhang, W., 2014. The Significance of "Object" in Cultural Heritage Digital Display, CHCD3rd International Symposium on Cultural Heritage Conservation and Digitization. 